\title{
The amazing diversity in the hot gas content of an X-ray unbiased massive galaxy clusters sample
}

\author{
S. Andreon ${ }^{1}$, Ana Laura Serra ${ }^{2}$, A. Moretti ${ }^{1}$, and G. Trinchieri ${ }^{1}$ \\ 1 INAF-Osservatorio Astronomico di Brera, via Brera 28, 20121 Milano, Italy \\ e-mail: stefano.andreon@brera.inaf.it \\ 2 Dip. di Fisica, Università degli Studi di Milano, via Celoria 16, 20133 Milano, Italy \\ Received 21 September 2015 / Accepted 10 November 2015
}

\section{ABSTRACT}

\begin{abstract}
We aim to determine the intrinsic variety, at a given mass, of the properties of the intracluster medium in clusters of galaxies. This requires a cluster sample selected independently of the intracluster medium content for which reliable masses and subsequent X-ray data can be obtained. We present one such sample, consisting of 34 galaxy clusters selected independently of their X-ray properties in the nearby $(0.050<z<0.135)$ Universe and mostly with $14<\log M_{500} / M_{\odot} \lesssim 14.5$, where masses are dynamically estimated. We collected the available X-ray observations from the archives and then observed the remaining clusters with the low-background Swift $\mathrm{X}$-ray telescope, which is extremely useful for sampling a cluster population expected to have low surface brightness. We found that clusters display a large range (up to a factor 50) in X-ray luminosities within $r_{500}$ at a given mass, whether or not the central emission $\left(r<0.15 r_{500}\right)$ is excised, unveiling a wider cluster population than seen in Sunayev-Zeldovich surveys or inferred from the population seen in X-ray surveys. The measured dispersion is $0.5 \mathrm{dex}$ in $L_{\mathrm{X}}$ at a given mass.
\end{abstract}

Key words. galaxies: clusters: intracluster medium - X-rays: galaxies: clusters - galaxies: clusters: general - methods: statistical

\section{Introduction}

The X-ray observations of the hot intracluster medium (ICM) of galaxy clusters provide quantities such as its mass, temperature $(T)$, and X-ray luminosity $\left(L_{X}\right)$. The analysis of the scaling relations between these physical quantities gives considerable insight into the physical processes occurring in the ICM (e.g., Rosati et al. 2002; Voit 2005). Scaling relations provide the basis for cosmological estimates based on galaxy clusters (e.g., Vikhlinin et al. 2009).

Scaling relations have generally been derived for X-ray selected samples. However, it is becoming generally appreciated (Pacaud et al. 2007; Stanek et al. 2006; Pratt et al. 2009; Andreon et al. 2011; Andreon \& Moretti 2011; Eckert et al. 2011; Planck Collaboration IX 2011; Planck Collaboration Int. 2012; Maughan et al. 2012) that X-ray selected clusters offer a biased view of the cluster population because, at a given mass, brighter-than-average clusters are easier to select and include in a sample, while fainter-than-average clusters are easily missed. In X-ray selected samples, the amplitude of the bias cannot be determined from the data (and selection function) alone, and input from non- $X$ ray selected samples is needed to break the degeneracy between the intercept and the scatter of the scaling relations. Alternatively, a strong prior on one of the parameters, for example based on numerical simulations, should be adopted (e.g., Vikhlinin et al. 2009). In spite of the interest on the subject and the general awareness of the biases induced by the X-ray selection, a sample of clusters observed in X-rays whose selection is, at a given mass, independent of the cluster ICM content is still missing in the literature. In this respect, Sunayev-Zeldovich (SZ) based surveys do not seem to represent an alternative, given that they also use the ICM to select the clusters, thus, they may miss clusters with low gas fractions.
Our current (and future) understanding of the complex physics involved in the cluster formation (see, e.g., Maughan et al. 2012), as well as the cosmological power of surveys, are severely affected by these systematics that can be understood only with the aid of cluster samples that are not X-ray selected. Future cosmological surveys (e.g., eROSITA), designed to supply large amounts of data from clusters, will benefit from an accurate understanding of the biases associated with the X-ray selection because the biases can only be understood with the aid of cluster samples that are not X-ray selected.

A population of clusters under-represented in X-ray selected samples have been already discovered among SZ-selected clusters (Planck Collaboration IX 2011; Planck Collaboration Int. 2012). The size of the missing population is not yet quantified; moreover, the SZ selection is not a satisfactorily alternative because being based on the ICM content it can miss clusters with low gas fractions. Andreon \& Moretti (2011) studied a colorselected cluster sample and found a large variety $(0.5 \mathrm{dex})$ of $\mathrm{X}$-ray luminosities at a given richness. Given that richness correlates tightly with mass (e.g., Andreon \& Hurn 2010; Andreon 2015), this suggests that there is a large variety in the $L_{X}$ at a given mass. So far, however, this is based on indirect evidence because the mass of the studied clusters has not been directly measured.

To firmly establish the variety of intracluster medium properties of galaxy clusters of a given mass and to break the degeneracies between the parameters of the X-ray cluster scaling relations is paramount to assemble cluster samples having three features: 1) observed in X-ray; 2) with known mass; and 3 ) whose selection is, at a given cluster mass, independent of the intracluster medium content. If masses are derived from the $\mathrm{X}$-ray data, then one should account for the covariance between 
Table 1. Cluster sample and results of the analysis.

\begin{tabular}{|c|c|c|c|c|c|c|c|c|c|c|c|c|c|c|c|}
\hline (1) & (2) & $\begin{array}{r}\text { Dec } \\
(3) \\
\end{array}$ & $\begin{array}{l}z \\
(4) \\
\end{array}$ & $\begin{array}{c}\log M_{500} \\
{\left[M_{\odot}\right]} \\
(5) \\
\end{array}$ & $\begin{array}{l}\text { err } \\
\text { dex } \\
(6) \\
\end{array}$ & $\begin{array}{l}N \\
\text { (7) } \\
\end{array}$ & $\begin{array}{c}\log M_{500} \\
{\left[M_{\odot}\right]} \\
(8) \\
\end{array}$ & $\begin{array}{c}\text { err } \\
\text { dex } \\
(9) \\
\end{array}$ & $(10)$ & $\begin{array}{c}\text { Telescope } \\
(11) \\
\end{array}$ & $\begin{array}{r}t_{\exp } \\
\mathrm{ks} \\
(12) \\
\end{array}$ & $\begin{array}{c}\log L_{\mathrm{X}} \\
\mathrm{erg} \mathrm{s}^{-1} \\
(13) \\
\end{array}$ & $\begin{array}{l}\text { err } \\
\text { dex } \\
(14) \\
\end{array}$ & $\begin{array}{c}\log L_{X} \\
\text { erg s}^{-1} \\
(15) \\
\end{array}$ & $\begin{array}{l}\text { err } \\
\text { dex } \\
(16) \\
\end{array}$ \\
\hline CL2081 & 0.167 & 14.550 & 0.093 & 14.39 & 0.09 & 94 & 13.94 & 0.28 & 11 & Swift & 9.5 & 42.44 & 0.11 & 42.09 & 0.19 \\
\hline CL2015 & 13.966 & -9.986 & 0.055 & 14.36 & 0.09 & 214 & 14.27 & 0.12 & 61 & Swift & 9.0 & 42.97 & 0.05 & 42.79 & 0.06 \\
\hline CL2045 & 22.887 & 0.556 & 0.079 & 13.86 & 0.10 & 66 & 13.95 & 0.16 & 45 & Swift & 10.1 & 43.20 & 0.03 & 43.11 & 0.04 \\
\hline CL2010 & 29.071 & 1.051 & 0.080 & 14.34 & 0.09 & 179 & 14.39 & 0.12 & 92 & Swift & 9.4 & 43.25 & 0.04 & 43.10 & 0.05 \\
\hline CL2007 & 46.572 & -0.140 & 0.109 & 14.28 & 0.09 & 164 & 14.22 & 0.14 & 57 & Swift & 9.1 & 42.84 & 0.09 & 42.68 & 0.12 \\
\hline CL3023 & 122.535 & 35.280 & 0.084 & 14.05 & 0.09 & 173 & 14.31 & 0.17 & 44 & Swift & 9.6 & 43.08 & 0.04 & 42.88 & 0.05 \\
\hline CL3030 & 126.371 & 47.130 & 0.127 & 14.52 & 0.15 & 364 & 14.67 & 0.07 & 156 & Swift & 16.5 & 44.05 & 0.01 & 43.93 & 0.02 \\
\hline CL3009 & 136.977 & 52.790 & 0.099 & 13.89 & 0.09 & 72 & 13.39 & 0.24 & 17 & Swift & 8.4 & 42.97 & 0.05 & 42.70 & 0.08 \\
\hline CL1209 & 149.161 & -0.358 & 0.087 & 14.03 & 0.09 & 115 & 14.29 & 0.15 & 43 & $X M M$ & 49.4 & 42.54 & 0.02 & 42.23 & 0.03 \\
\hline CL3053 & 160.254 & 58.290 & 0.073 & 13.52 & 0.27 & 99 & 13.63 & 0.20 & 31 & Swift & 14.4 & 42.61 & 0.08 & 42.48 & 0.07 \\
\hline CL3000 & 163.402 & 54.870 & 0.072 & 14.58 & 0.09 & 180 & 14.18 & 0.15 & 59 & Swift & 8.8 & 43.51 & 0.03 & 43.35 & 0.05 \\
\hline \multirow[t]{2}{*}{ CL3046 } & 164.599 & 56.790 & 0.135 & 14.62 & 0.09 & 172 & 14.63 & 0.15 & 104 & Swift & 9.0 & 44.46 & 0.01 & 44.37 & 0.01 \\
\hline & & & & & & & & & & Chandra & 7.7 & 44.48 & 0.01 & 44.37 & 0.01 \\
\hline CL1033 & 167.747 & 1.128 & 0.097 & 13.83 & 0.12 & 77 & 13.79 & 0.21 & 20 & Swift & 9.0 & 43.15 & 0.04 & 43.05 & 0.05 \\
\hline \multirow[t]{2}{*}{ CL1073 } & 170.726 & 1.114 & 0.074 & 14.20 & 0.09 & 149 & 14.15 & 0.12 & 63 & Chandra & 21.7 & 43.17 & 0.03 & 43.10 & 0.03 \\
\hline & & & & & & & & & & Swift & 14.6 & 43.03 & 0.05 & 42.95 & 0.06 \\
\hline CL3013 & 173.311 & 66.380 & 0.115 & 14.28 & 0.13 & 148 & 14.29 & 0.12 & 111 & Swift & 9.7 & 44.05 & 0.01 & 43.89 & 0.02 \\
\hline CL1014 & 175.299 & 5.735 & 0.098 & 14.40 & 0.09 & 120 & 14.40 & 0.16 & 51 & Swift & 12.0 & 43.47 & 0.03 & 43.31 & 0.04 \\
\hline CL1020 & 176.028 & 5.798 & 0.103 & 13.94 & 0.35 & 29 & 14.61 & 0.14 & 43 & Swift & 6.8 & 43.74 & 0.02 & 43.61 & 0.03 \\
\hline CL1038 & 179.379 & 5.098 & 0.076 & 14.21 & 0.09 & 95 & 14.28 & 0.13 & 54 & Swift & 8.7 & 43.43 & 0.03 & 43.37 & 0.03 \\
\hline CL1015 & 182.570 & 5.386 & 0.077 & 13.85 & 0.19 & 79 & 14.14 & 0.15 & 37 & Swift & 17.3 & 43.65 & 0.01 & 43.55 & 0.01 \\
\hline CL1120 & 188.611 & 4.056 & 0.085 & 14.02 & 0.09 & 50 & 14.33 & 0.21 & 25 & Swift & 10.9 & 42.63 & 0.09 & 42.50 & 0.11 \\
\hline CL1041 & 194.673 & -1.761 & 0.084 & 14.31 & 0.09 & 370 & 14.58 & 0.11 & 153 & Chandra & 38.2 & 44.39 & 0.01 & 44.17 & 0.01 \\
\hline CL1132 & 195.143 & -2.134 & 0.085 & 13.79 & 0.24 & 42 & 13.70 & 0.17 & 27 & Swift & 9.6 & 42.34 & 0.10 & 42.04 & 0.15 \\
\hline CL1052 & 195.719 & -2.516 & 0.083 & 14.31 & 0.09 & 262 & 14.57 & 0.10 & 96 & $X M M$ & 49.5 & 43.65 & 0.01 & 43.39 & 0.01 \\
\hline CL1009 & 198.057 & -0.974 & 0.085 & 14.00 & 0.26 & 236 & 14.28 & 0.12 & 56 & Chandra & 20.9 & 43.38 & 0.01 & 43.24 & 0.02 \\
\hline CL1022 & 199.821 & -0.919 & 0.083 & 13.86 & 0.12 & 87 & 13.56 & 0.16 & 36 & $X M M$ & 31.2 & 42.55 & 0.10 & 42.55 & 0.10 \\
\hline CL3049 & 203.264 & 60.120 & 0.072 & 13.63 & 0.10 & 49 & 13.86 & 0.23 & 32 & Swift & 12.3 & 43.09 & 0.03 & 42.97 & 0.03 \\
\hline CL1030 & 206.165 & 2.860 & 0.078 & 14.16 & 0.09 & 86 & 14.12 & 0.21 & 41 & Swift & 13.1 & 42.75 & 0.06 & 42.54 & 0.09 \\
\hline CL1001 & 208.256 & 5.134 & 0.079 & 14.30 & 0.11 & 171 & 14.57 & 0.09 & 124 & Swift & 31.0 & 43.87 & 0.01 & 43.79 & 0.01 \\
\hline CL1067 & 212.022 & 5.418 & 0.088 & 13.92 & 0.38 & 57 & 14.29 & 0.14 & 42 & Swift & 10.9 & 43.33 & 0.04 & 43.25 & 0.04 \\
\hline CL1018 & 214.398 & 2.053 & 0.054 & 13.68 & 0.24 & 123 & 14.27 & 0.10 & 79 & Swift & 9.7 & 42.83 & 0.04 & 42.73 & 0.05 \\
\hline CL1011 & 227.107 & -0.266 & 0.091 & 14.23 & 0.17 & 48 & 14.46 & 0.14 & 42 & Swift & 13.7 & 42.96 & 0.05 & 42.77 & 0.07 \\
\hline CL1039 & 228.809 & 4.386 & 0.098 & 14.32 & 0.16 & 76 & 14.63 & 0.13 & 68 & $X M M$ & 11.0 & 43.52 & 0.02 & 43.42 & 0.02 \\
\hline \multirow[t]{2}{*}{ CL1047 } & 229.184 & -0.969 & 0.118 & 14.01 & 0.23 & 121 & 13.81 & 0.23 & 28 & Swift & 8.8 & 43.71 & 0.03 & 43.63 & 0.04 \\
\hline & & & & & & & & & & $X M M$ & 39.6 & 43.78 & 0.01 & 43.70 & 0.01 \\
\hline CL3020 & 232.311 & 52.860 & 0.073 & 14.12 & 0.14 & 81 & 14.18 & 0.18 & 53 & Swift & 7.3 & 43.30 & 0.04 & 43.20 & 0.04 \\
\hline
\end{tabular}

Notes. The table lists: cluster id (Col. 1); coordinates (Cols. 2 and 3); redshift $z$ (Col. 4); caustic masses $M_{500}$ (Cols. 5 and 6); number of galaxies within the caustics (Col. 7); velocity-dispersion based masses $M_{500}$ (Cols. 8 and 9); number of galaxies within $r_{200}$ and $\left|\Delta_{v}\right|<3500 \mathrm{~km} \mathrm{~s}{ }^{-1}$ (Col. 10); X-ray telescope used (Col. 11); effective exposure time (Col. 12); X-ray luminosity $L_{X}\left(<r_{500}\right)$ in the $[0.5$, 2] keV band (Cols. 13 and 14); core-excised X-ray luminosity $L_{\mathrm{X}}\left(0.1 r_{500}<r<r_{500}\right)$ in the $[0.5,2] \mathrm{keV}$ band (Cols. 15 and 16).

$\mathrm{X}$-ray luminosity and mass induced by the double use of the same photons.

The purpose of this work is to build, observe, and analyze one such cluster sample. To this end, in 2011 our team started an observational program with the X-ray telescope on board of Swift, dubbed X-ray unbiased cluster survey (XUCS). With this program, we were able to uncover a wider range in luminosities relatively to what expected on the basis of the Representative XMM-Newton Cluster Structure survey (REXCESS), or even Planck selected clusters. The impatient reader may want to quickly browse Sect. 2 (sample selection), read Sect. 3 (which illustrates the main conclusion of the paper with an example), skip the technical analysis in Sect. 4, and read the results based on the whole sample in Sect. 5.

Throughout this paper, we assume $\Omega_{\mathrm{M}}=0.3, \Omega_{\Lambda}=0.7$, and $H_{0}=70 \mathrm{~km} \mathrm{~s}^{-1} \mathrm{Mpc}^{-1}$. Results of stochastic computations are given in the form $x \pm y$, where $x$ and $y$ are the posterior mean and standard deviation. The latter also corresponds to $68 \%$ intervals because we only summarize posteriors close to Gaussian in this way.

\section{Sample selection}

Our sample, named XUCS, consists of 34 clusters in the very nearby universe $(0.050<z<0.135)$ extracted from the $\mathrm{C} 4$ cata$\log$ (Miller et al. 2005) in regions of low Galactic absorption.
The C4 catalog identifies clusters as overdensities in the local Universe in a seven-dimensional space (position, redshift, and colors; see Miller et al. 2005, for details) using SDSS data (Abazajian et al. 2004). Among all C4 high-quality clusters (>30 spectroscopic members within a radius of $1.5 \mathrm{Mpc}, 55$ on average) with a (dynamical) mass $\log M>14.2 M_{\odot}$ (derived from the $\mathrm{C} 4 \sigma_{v}>500 \mathrm{~km} \mathrm{~s}^{-1}$ ), we selected the 34 nearest (to minimize exposure times) which are also smaller than the Swift XRT field of view ( $r_{500} \lesssim 9$ arcmin).

The XUCS cluster sample is listed in Table 1. There is no $\mathrm{X}$-ray selection in our sample, meaning that 1) the probability of inclusion of the cluster in the sample is independent of its X-ray luminosity (or count rate); and that 2) no cluster is kept or removed on the basis of its X-ray properties ${ }^{1}$.

As detailed in Table 2, many of our sample clusters were already detected by the previous generation of X-ray telescopes and surveys (e.g., REFLEX and NORAS; Bohringer et al. 2000, 2004), some are listed in the Abell et al. (1989) catalog and

1 Our original program targeted a slightly larger cluster sample. However, because of the approximation in the coordinates listed in the optical C4 catalog, the center of a few clusters fell too close to XRT field-of-view boundaries, or in some cases, was missed altogether. Furthermore, the dynamical analysis of two of the original clusters shows that they are two parts of the same dynamical entity at the $r_{200}$ scale, making the derived masses of the individual clusters unreliable. For this reason, we removed these two targets from the sample. 
Table 2. Literature names.

\begin{tabular}{|c|c|}
\hline Id & Literature names \\
\hline \multicolumn{2}{|l|}{ CL2081 } \\
\hline CL2005 & A117, PSZ2G126.72-72.82 \\
\hline CL2045 & A208, RXC J0131.7+0033, MCXCJ0131.7+0033 \\
\hline CL2010 & A279 \\
\hline CL2007 & A412 \\
\hline CL3023 & A628 \\
\hline CL3030 & A655, RXC J0825.5+4707, MCXCJ0825.5+4707, PSZ2G172.63+35.15 \\
\hline CL3009 & XCLASS 1943 \\
\hline \multicolumn{2}{|l|}{ CL1209 } \\
\hline \multicolumn{2}{|l|}{ CL3053 } \\
\hline CL3000 & RXC J1053.7+5452, MCXCJ1053.7+5452 \\
\hline CL3046 & A1132, МCXCJ1058.4+5647, PSZ2G149.22+54.18 \\
\hline CL1033 & A1189 \\
\hline CL1073 & A1238, RXC J1122.8+0106, MCXCJ1122.8+0106 \\
\hline CL3013 & A1302, MCXCJ1133.2+6622, PSZ2G134.70+48.91 \\
\hline CL1014 & A1346, PSZ2G261.88+62.85 \\
\hline \multicolumn{2}{|l|}{ CL1020 } \\
\hline CL1038 & A1424, MCXCJ1157.4+0503 \\
\hline CL1015 & ZWCL1207.5+0542, RXC J1210.3+0523, MCXCJ1210.3+0523 \\
\hline \multicolumn{2}{|r|}{ 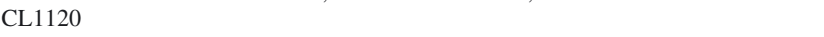 } \\
\hline CL1041 & $\begin{array}{l}\text { A1650, RXC J1258.6-0145, MCXCJ1258.6-0145, } \\
\text { Hydra, PSZ2G306.66+61.06 }\end{array}$ \\
\hline \multicolumn{2}{|r|}{ 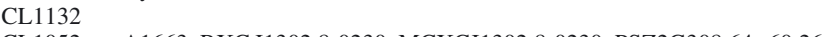 } \\
\hline CL1052 & A1663, RXC J1302.8-0230, MCXCJ1302.8-0230, PSZ2G308.64+60.26 \\
\hline CL1009 & A1692, RXJ1218.9+0515 \\
\hline CL1022 & 2PI0.084J1319.3-0055 \\
\hline \multicolumn{2}{|l|}{ CL3049 } \\
\hline CL1030 & A1780 \\
\hline CL1001 & A1809, MCXCJ1353.0+0509, PSZ2G339.47+63.56 \\
\hline CL1067 & A1864 \\
\hline CL1018 & MKW6 \\
\hline CL1011 & A2026, XMMXCSJ1508.4-0015 \\
\hline CL1039 & A2048 \\
\hline CL1047 & A2051, RXC J1516.5-0056, MCXCJ1516.5-0056, PSZ2G000.04+45.13 \\
\hline CL3020 & \\
\hline
\end{tabular}

some are also in the Planck cluster catalog (Planck Collaboration XXVII 2015). This information, not used to select the sample, is given for completeness and it also illustrates that the clusters selected are real structures present in other catalogs.

We collected the few X-ray observations present in the XMM-Newton or Chandra archives and we observed 28 clusters with Swift (individual exposure times between 9 and $31 \mathrm{ks}$, total exposure time $\sim 0.35 \mathrm{Ms}$, see Table 1). Swift has the advantage of a low X-ray background (Moretti et al. 2009), making it extremely useful for sampling a cluster population expected to have low surface brightness (Andreon \& Moretti 2011).

\section{The straightforward comparison}

To illustrate the diversity in ICM properties of clusters of a given mass in the simplest possible way, we consider two clusters, CL3013 and CL2007. We chose these two clusters because they have identical masses $\left(\log M_{500} / M_{\odot}=14.28\right)$ and velocity dispersions (600 $\mathrm{km} \mathrm{s}^{-1}$; see Fig. 1); very similar redshifts $(z=0.115$ and 0.109$)$; similar number of galaxies within the caustics $(\sim 150)$; and X-ray data of the same depth $(\sim 9.5 \mathrm{ks}$ with Swift XRT; see Table 1) in directions having the same Galactic absorption. The Swift XRT images shown in Fig. 2 indicate that the X-ray emission of the two clusters is vastly different. We collected 1027 net photons in [0.5-2] keV band for CL3013, and 65 for CL2007 within $0.83 \mathrm{Mpc}$ (corresponding to the $r_{500}$ value). The more detailed analysis presented in Sect. 4.1 confirms the large (a factor $\sim 16$ ) difference in luminosities (total and coreexcised) and that the difference is not due to a cool core in CL3013 because the latter has a $\sim 10$ times larger count rate at all radii.

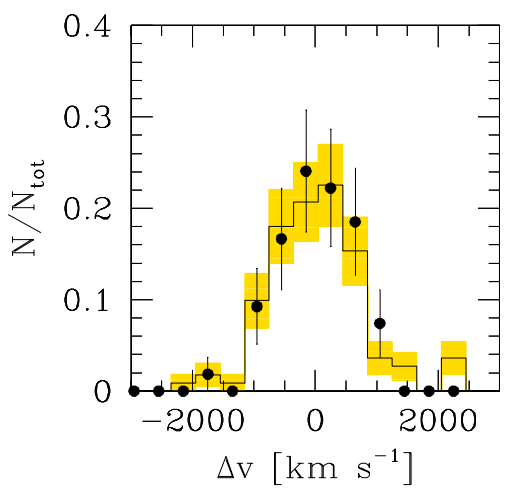

Fig. 1. Velocity distribution of the galaxies in clusters CL3013 (histogram) and CL2007 (points). Error-bars to the histogram are shown with the shading. In this purely illustrative figure, error-bars are simply $\sqrt{n}$-based.

As discussed in detail in Sect. 5, CL3013 and CL2007 differ too much in X-ray luminosity to obey the low-scatter scaling relation of X-ray selected cluster samples, such as the Representative XMM-Newton Cluster Structure survey (REXCESS) sample (Bohringer et al. 2007), even after taking the X-ray selection of the latter into account. This is the main result of this work, which we now illustrate for the full sample.

\section{Collective analysis of the full sample}

In the following, we describe the analysis procedures to compute the mass and X-ray luminosity in the [0.5-2] keV band of the clusters of our sample.

\subsection{X-ray analysis}

A few clusters of our sample were observed with XMM-Newton (EPIC-MOS1 and 2; Turner et al. 2001) and Chandra ACIS-I (Garmire et al. 2003; see Table 1 for details ${ }^{2}$ ), which also reports effective exposure times (after flares cleaning). The remaining clusters were observed with the X-ray telescope (XRT) on board the Swift satellite (Gehrels et al. 2004) ${ }^{3}$. We reduced the X-ray data using the standard data reduction procedures (Moretti et al. 2009; $\mathrm{XMMSAS}^{4}$ or $\mathrm{CIAO}^{5}$ ).

As shown in Figs. 2 and 3, clusters in our sample show a variety of X-ray morphologies. CL3000 shows an offset between the galaxy number density distribution and the X-ray emission (see Fig. 3), a bit like the Bullet cluster (Clowe et al. 2004). CL1022 is a bimodal cluster formed by two distinct clumps of X-ray emitting gas and galaxies (each one peaked on its own brightest cluster galaxy; BCG hereafter) of comparable richness and $\mathrm{X}$-ray luminosity. The other clusters show a range of morphologies from regular (CL1209, see Fig. 3) to bimodal/irregulars, as evaluated from the available number of photons (the median is

2 XMM obsid 0206430101 (PI C. Miller), 0201751301 (PI. P. Mazzotta), 0201902101 (PI H. Boheringer), and 0653810601 (PI Ming Sun); Chandra obsid 4990 and 4991 (PI Bower), 5823 (PI Donahue), and 13376 (PI Murray).

3 Swift proposals id 8110004, 9120007, and 1013012 (PI S. Andreon), about one third of them executed on Italian time.

4 http://xmm.esac.esa.int/sas

5 http://cxc.harvard.edu/ciao/ 


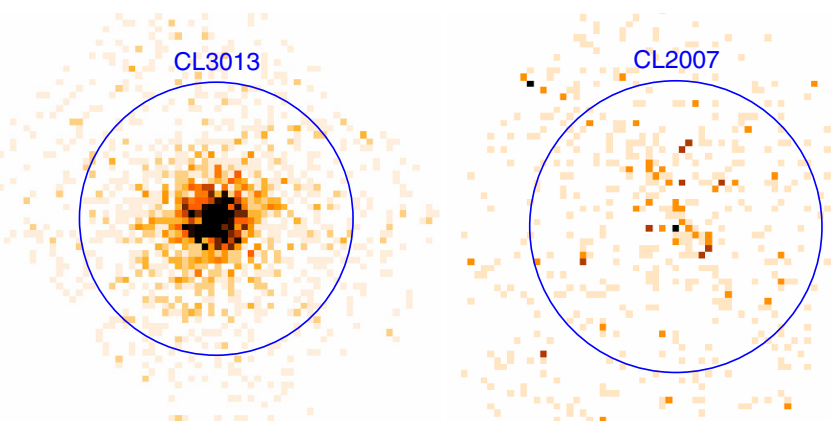

Fig. 2. Binned X-ray image of CL3013 and CL2007 in the [0.5-2] keV band observed for about $9.5 \mathrm{ks}$ each with XRT on Swift. These clusters have identical masses, velocity dispersions, and redshift, but X-ray luminosities differing by a factor 15 . The circle shows the $0.83 \mathrm{Mpc}$ radius (the $r_{500}$ value).

400 photons within $0.15 r_{500}<r<r_{500}$ and 550 within $r<r_{500}$ in the [0.5-2] keV band) ${ }^{6}$.

Point sources are detected by a wave detection algorithm, and we masked pixels affected by them when calculating radial profiles and fluxes. In our analysis, we used exposure maps to calculate the effective exposure time accounting for dithering, vignetting, CCD defects, gaps, and excised regions.

Since Swift observations are taken with different roll angles and sometimes different pointing centers, the exposure map may show large differences at very large off-axis angles. To avoid regions of too low exposure, we only consider regions where the exposure time is larger than $50 \%$ of the central value. Furthermore, we truncate the radial profile when one-third of the circumference is outside the $50 \%$ exposure region defined above. We take the position of the BCG closest to the X-ray peak as cluster center (the northern one in case of bimodal cluster CL1022).

To measure the surface brightness profile, we extract the distance from the cluster center $r$ of individual photon in the [0.5-2] keV band, and we individually fit them (i.e., without any radial binning, as detailed in Andreon et al. 2008, 2011; a similar approach is followed in CIAO-Sherpa), with the following generalized $\beta$ model adapted from Vikhlinin et al. (2006):

$S(r) \propto S_{0}\left(r / r_{\mathrm{c}}\right)^{-\alpha}\left(1+\left(r / r_{\mathrm{c}}\right)^{2}\right)^{-3 \beta+0.5+\alpha / 2}+C$,

where $S_{0}, r_{\mathrm{c}}$, and $C$ are the central surface brightness, core radius, and the background constant, respectively. The $\alpha$ parameter models the power-law-type cusp typical of cool-core clusters and is a parameter requested by the data of some of our clusters. Chandra data also require us to excise the inner 3-5 arcsec region because of the BCG emission. Our fit accounts for vignetting, excised regions, background level, variation in exposure time, and Poisson fluctuations using the likelihood in Andreon et al. (2008). The parameter $\beta$ is fixed at $2 / 3$ because our data cannot constrain all parameters for all clusters. For the other parameters, we took weak priors, and, in particular, we took a uniform prior for $S_{0}$ and $C$, constrained to be positive (to avoid unphysical values), and a Gaussian prior on $r_{\mathrm{c}}$ with the center equal to $r_{500} / 5$ (suggested by Ettori et al. 2015), with a $30 \%$ sigma (i.e., $r_{\mathrm{c}} \in\left(0, r_{500}\right)$ with $99 \%$ probability). A posteriori, all (posterior mean) $r_{\mathrm{c}}$ are well within this range.

\footnotetext{
6 The radius $r_{\Delta}$ is the radius within which the enclosed average mass density is $\Delta$ times the critical density at the cluster redshift. It is computed during the dynamical analysis in Sect. 4.2.2.
}

As an example, Fig. 4 shows the observed surface brightness profile, the fitted generalized beta model, and its $68 \%$ uncertainty for the two clusters shown in Fig. 2. CL3013 has no cool core and a 10 times larger count rate than CL 2007, at all radii, as mentioned in Sect. 3 .

By integrating the fitted model within the ranges $0<r<r_{500}$ and $0.15 r_{500}<r<r_{500}$ and assuming a $3.5 \mathrm{keV}$ temperature, we obtain the X-ray luminosity within $r_{500}, L_{500}=L_{X}\left(<r_{500}\right)$, and the core-excised luminosity, $L_{500, \mathrm{ce}}=L_{\mathrm{X}}\left(0.15<r / r_{500}<1\right)$, listed in Table 1 . Adopting $k T=8 \mathrm{keV}$ would only change the derived luminosities by 0.01 dex. This ensures that our conclusions below are not resulting from temperature variations that we have neglected across the sample. The smallest $0.15 r_{500}$ radius is 49 arcsec, much larger than PSF of the used instruments ( $\lesssim 8$ arcsec), so that the contribution of any cool-core flux spilled out of the excised region is negligible.

For all but two clusters $r_{500}$ is smaller than the radius of the (conservatively taken) last point in the surface brightness profile, $r_{\text {last }}$. For CL2015 and CL3000 it is marginally outside it and we measure the extrapolation based on the best-fit model to be 6 to $8 \%$. The error on the uncertain extrapolation is automatically accounted for by our $L_{X}$ computation (via Eq. (1)).

Although our radial profile model is very flexible in describing the surface brightness profile of clusters, it assumes a unimodal X-ray emission and, therefore, cannot deal with the bimodal CL1022 cluster. For this cluster, the X-ray luminosity is derived by simple aperture photometry centering the aperture on the northern clump (and ignoring gaps, regions unobserved because of other sources, variation in exposure time, etc.). As a check, we derive the X-ray luminosities of CL1132 and CL1015 in a similar way, i.e., by simply counting photons in the image and with the same limitations as before. We found results consistent with those derived by fitting a radial profile, which provides more reliable $L_{X}$ errors however.

Three clusters have data from two X-ray telescopes and we compared the resulting luminosities from the different instruments. CL3046 has identical luminosities, the other two clusters differ by an amount that is negligible for our purposes ( $~ 0.1$ dex). Similarly, the luminosities within the REXCESS (Pratt et al. 2009) $r_{500}$ of the two clusters in common with REXCESS are consistent.

\subsection{Dynamical analysis}

Data for the dynamical analysis are drawn from the 12th SDSS data release (Alam et al. 2015), Galaxy And Mass Assembly (Liske et al. 2015; Baldry et al. 2014), 2dF (Colless et al. 2001), $6 \mathrm{dF}$ (Jones et al. 2009), Rines et al. (2013), Hill \& Oegerle (1993), Miller et al. (2002), and Pimbblet et al. (2006). Both velocity dispersion and caustics analyses below assume the center fixed at the location of the BCG closest to the X-ray peak.

\subsubsection{Velocity dispersion based masses}

The determination of a dispersion in presence of interlopers/outliers/background is a well-known problem with a long history: Beers et al. (1990) reduced the impact of the contaminating population by using lower weights for objects in the wings of the distribution. Andreon et al. (2008), Wojtak et al. (2011), and Andreon \& Waever (2015) modeled the probability that an object belongs to the interesting, or contaminating, population based on relative velocity from the barycenter, which improves the velocity dispersion estimates upon previous 

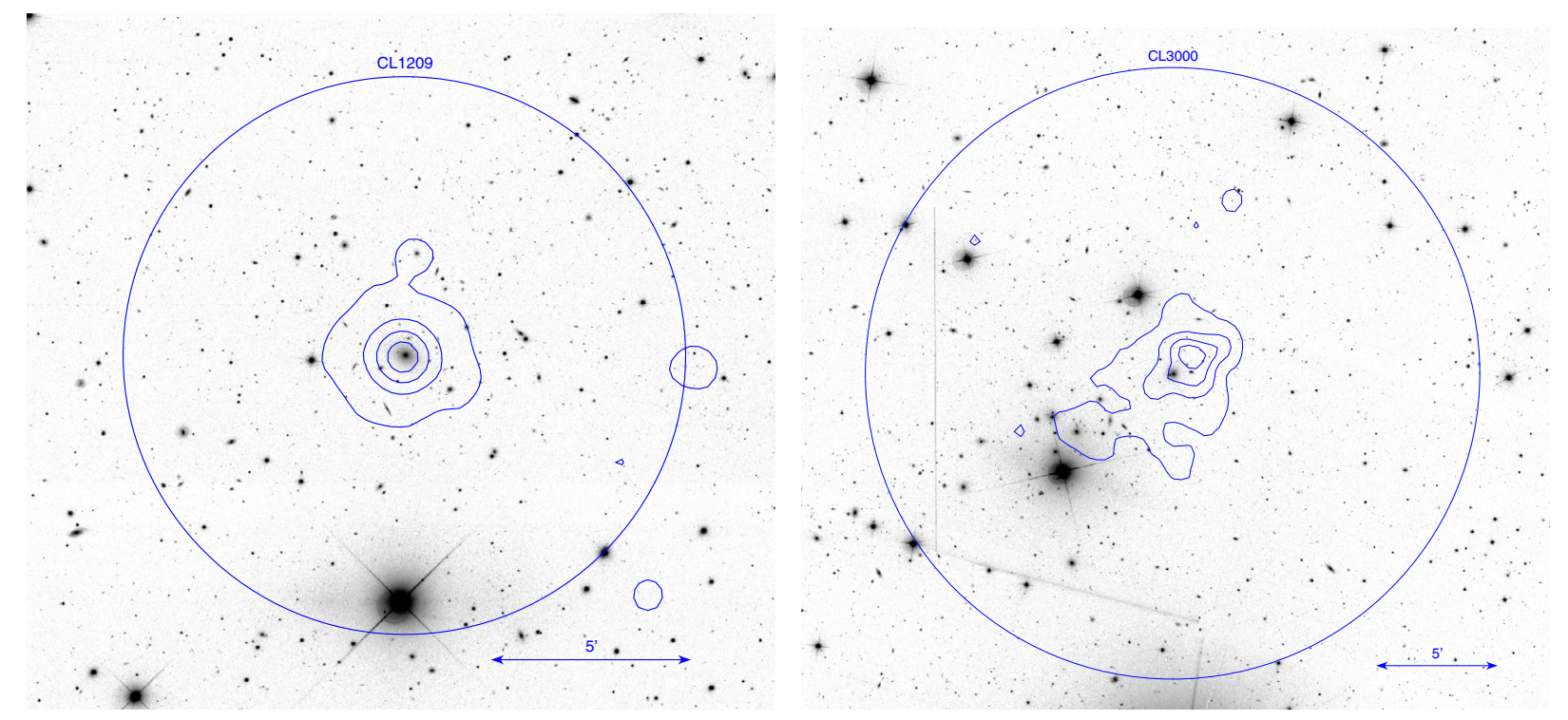

Fig. 3. Optical ( $r$-band) image of CL1209 (left-hand panel) and CL3000 (right-hand panel) with superimposed X-ray countours in the [0.5-2] keV band, the latter smoothed with a Gaussian with $\sigma \sim 45$ arcsec. The two clusters are among the X-ray faintest for their mass. The circle radius is equal to $r_{500}$. North is up, east is to the left.
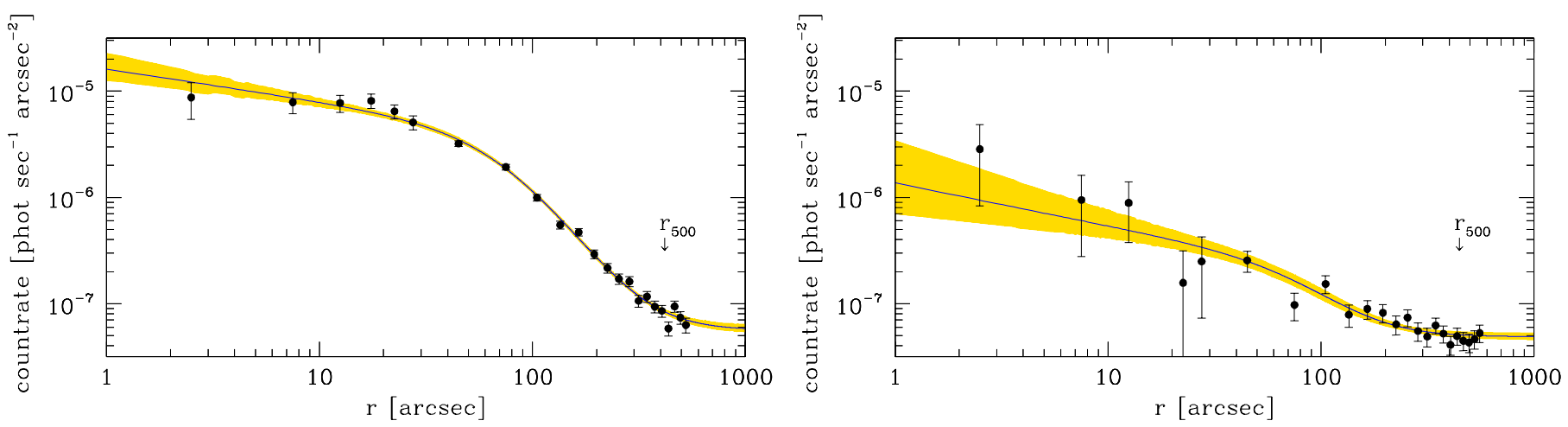

Fig. 4. X-ray radial profile ([0.5-2] keV band) of the clusters CL3013 (left-hand panel) and CL2007 (right-hand panel). The arrow marks the $r_{500}$ value.

approaches (Andreon et al. 2008; Andreon 2010). Here, we further refine the method with the full cluster centric distance information (i.e., not just the information that the galaxy is inside the considered radial range) in addition to velocity information.

First, we only consider galaxies within $\Delta v<3500 \mathrm{~km} \mathrm{~s}^{-1}$ and $r<r_{200}$ because outside this range galaxies are unlikely to be virialized cluster members. Second, following previous works, we model the distribution in the velocity space as a sum of a Gaussian (for cluster members) and a uniform distribution (for foreground/background galaxies) zeroed outside $\Delta v=3500 \mathrm{~km} \mathrm{~s}^{-1}$ because we discard galaxies with $\Delta v>$ $3500 \mathrm{~km} \mathrm{~s}^{-1}$. Third, we model the distribution in the radial direction as a King profile (for cluster members) plus a constant (for foreground/background galaxies), zeroed at $r>r_{200}$ because galaxies at larger radii are discarded from the sample. By fitting a radial profile, we improve upon previous approaches because we use the radial information in full. The radius $r_{200}$ is, however, unknown. We derive it through simple iterations; we first adopt a radius of $20 \mathrm{arcmin}$, and we derive $\sigma_{v}$ from galaxies within this region, then we convert $\sigma_{v}$ in $M_{200}$ using the $\sigma_{v}-M_{200}$ relation in Evrard et al. (2008). Since $r_{200} \propto M_{200}^{1 / 3}$, we then select only galaxies within this $r_{200}$, and repeat the procedure two more times. Convergence is already achieved at the first iteration.

We fit the spatial-velocity model to the (unbinned) position and velocity data by means of a Marcov-chain Monte-Carlo sampler. We adopt uniform prior on parameters, zeroed outside the physically acceptable range. Table 1 lists the derived cluster mass (obtained from the measured $\sigma_{v}$ using the $M_{200}-\sigma_{v}$ in Evrard et al. 2008, and then in $M_{500}$ assuming a Navarro et al. 1997 profile with concentration $c=5$ ), the number of fitted galaxies, and velocity barycenter. With $c=3$, masses would differ by about 0.04 dex, a difference negligible compared to our errors. The median number of fitted galaxies (positions and velocities) within $r_{200}$ is 51 , the interquartile range is 32 . The median mass of the cluster sample is $\log M_{500} / M_{\odot}$ is 14.2 , the interquartile range is 0.4 dex. As mentioned, the probabilistic approach offers more precise measurements and more robust estimates of the uncertainties than older approaches basically because more information in the data is used.

For a few clusters the assumption of a background population of galaxies uniform in velocity is not satisfied by the data because of the presence of velocity-coherent structures (a filament 

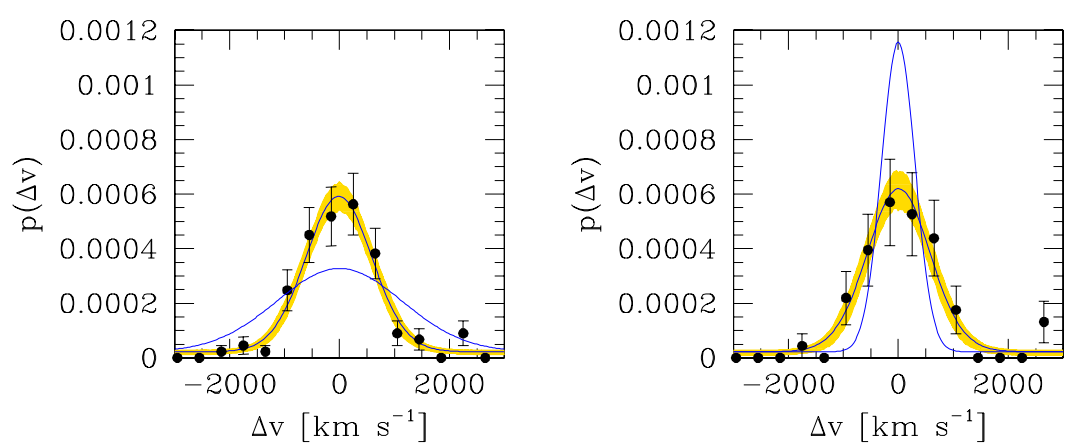

Fig. 5. Velocity distribution of the galaxies in the clusters CL3013 (left-hand panel) and CL2007 (right-hand panel). The solid line is the mean model fitted to the individual galaxy data in the $r-v$ plane, while the shading indicates the $68 \%$ uncertainty (highest posterior density interval). Points and approximated error bars are derived by ignoring the radial information, binning galaxies in velocity bins, and adopting approximated Poisson errors, as is commonly found in the literature. The blue curves shows the distribution appropriate for their observed X-ray luminosity (see Sect. 5.4 for details).

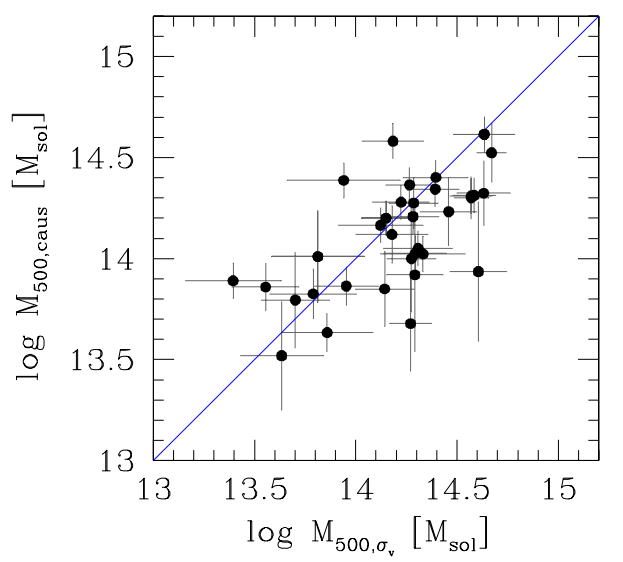

Fig. 6. Caustic masses (ordinate) vs. velocity dispersion based masses (abscissa). The blue line indicates equality and it is not a fit to the data.

or a group). In these cases, we narrowed the radial and/or velocity ranges to make our assumption valid. The caustic analysis described below and an attentive inspection of the space-phase diagram is quite useful to recognize these structures.

Figure 5 shows the observed data, the fitted model, and its $68 \%$ uncertainty for the two clusters in Figs. 1, 2, and 4.

\subsubsection{Caustics masses}

Caustic masses within $r_{200}, M_{200}$, have been derived following Diaferio \& Geller (1997), Diaferio (1999) and Serra et al. (2011). Basically, the caustic technique performs a measurement of the line-of-sight escape velocity and has the advantage of not assuming virial equilibrium, assumed instead when estimating velocity-dispersion-based masses. It only uses redshift and position of the galaxies to identify the caustics on the redshift diagram (clustrocentric distance vs. line-of-sight velocity), whose amplitude is a measure of the escape velocity of the cluster. The mass of a spherical shell within the radius $r$ is just the integral of the square of the caustic amplitude times the filling factor $F$, taken to be 0.5 in agreement with Diaferio \& Geller (1997) and Diaferio (1999). A byproduct of the caustic technique is the construction of a binary tree that arranges the galaxies hierarchically, according to their binding energy. This tool $^{7}$ is useful for recognizing possible structures in the considered field. To force convergence on the cluster under study (instead of a nearby but distinct cluster in the field), we fixed the cluster center at the optical/X-ray center.

Table 1 lists the cluster masses $M_{500}$, derived from the caustic $M_{200}$ by assuming a Navarro et al. (1997) profile with concentration $c=5$, as for $\sigma_{v}$-based masses. To account for systematic errors, mostly due to the intrinsic triaxial structure of halos, we adopt as mass error the maximum between $20 \%$ and the statistical error (Serra et al. 2011; Gifford \& Miller 2013). The median number of members within the caustics is 116 and the interquartile range is 45 . The median mass of the cluster sample is $\log M_{500} / M_{\odot}$ is 14.2 and the interquartile range is 0.4 dex.

Eight XUCS clusters have caustic masses also derived in Rines et al. (2006; 2013). Six out of eight clusters have masses in agreement at $1 \sigma$ level or better, the remaining two clusters at $\sim 1.5 \sigma$.

While positions and velocities are used by both the caustic analysis and velocity dispersion determinations, their use is very different. For example, the dynamical analysis uses galaxies within $r_{200}$, while this restriction does not apply to the caustic technique, which instead mostly uses galaxies at $r \gtrsim r_{200}$ (and the two $r_{200}$ are not necessarily the same). Furthermore, while we assume a uniform distribution in velocity space when we derive masses from the velocity dispersion, the caustic technique does not make this assumption.

Masses inferred from velocity dispersions agree extremely well with those inferred from caustics (see Fig. 6). The mean $\chi^{2}$ is 1.3 and the average scatter is 1.1 times the value expected based on the quoted errors. Furthermore, we understand the reason for the difference of the masses of the two clusters with the largest differences $(\sim 2 \sigma)$ : CL3000 is likely to have underestimated caustic errors and the velocity dispersion of CL1018 is likely overestimated because of a possible background velocity structure not accounted for.

\section{X-ray luminosity vs. masses}

The left-hand panels of Fig. 7 show the X-ray luminosity $L_{500}$ (bottom panels) and the core-excised X-ray luminosity $L_{500 \text {,ce }}$ (top panels) vs. mass for our cluster sample.

7 CausticApp is publicy available at http://personalpages.to. infn.it/ serra/causticapp.html 


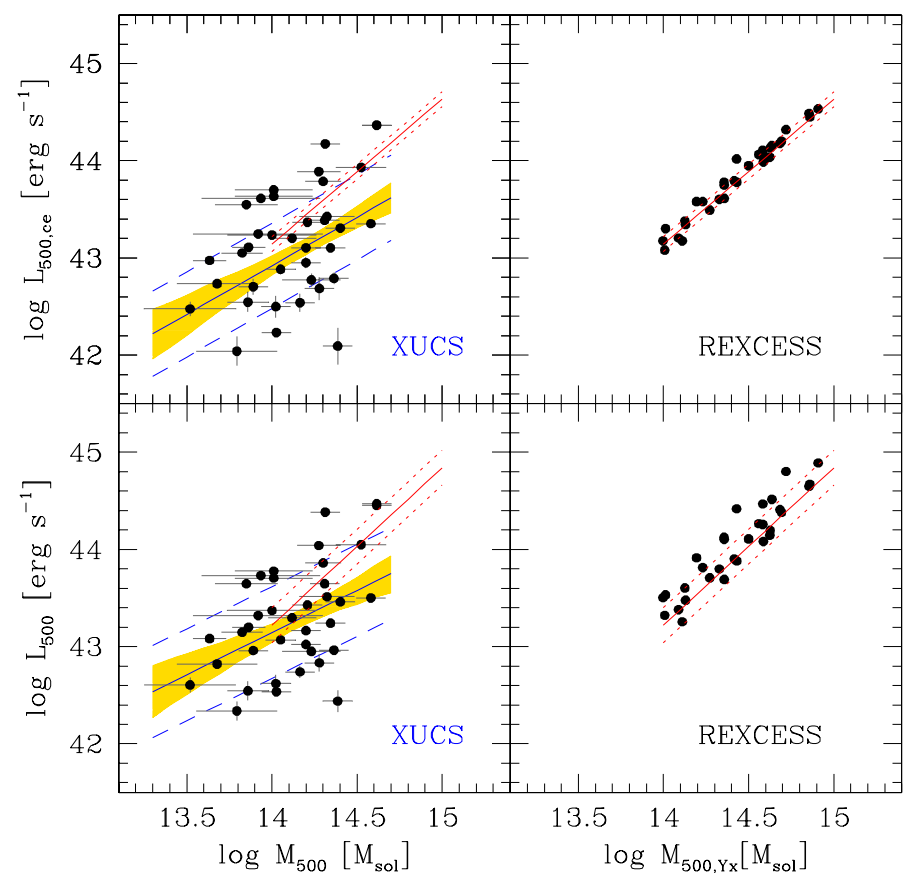

Fig. 7. X-ray luminosity ([0.5-2] keV band) vs. Mass. Top panels: core-excised. Bottom panels: non-core excised. Left-hand panels: our (XUCS) sample. Right-hand panels: REXCESS sample. The solid (red) and dotted lines are the REXCESS Malmquist-bias corrected mean and estimated scatter. The solid blue line surronded by a yellow shading indicates our fit to our data. The shading indicates its $68 \%$ uncertainty and the dashed blue dashed lines indicate the fit model \pm the intrinsic scatter.

We fit the data above with a linear relation with intrinsic scatter and errors ${ }^{8}$ with the JAGS Marcov-chain Monte-Carlo sampler, which adopts weak priors on parameters. For coreexcised luminosities we found

$\log L_{500, \mathrm{ce}}=(0.82 \pm 0.33)\left(\log M_{500} / M_{\odot}-14.2\right)+43.2 \pm 0.1$,

with an intrinsic scatter of $0.50 \pm 0.07$, and a very similar result for total luminosities

$\log L_{500}=(0.85 \pm 0.31)\left(\log M_{500} / M_{\odot}-14.2\right)+43.3 \pm 0.1$,

with an intrinsic scatter of $0.47 \pm 0.07$. Luminosities are expressed in erg s $\mathrm{s}^{-1}$ and the scatter accounts for both the noisiness of mass and X-ray luminosity. These results are robust to the modeling of the scatter: we find indistinguishable results by replacing the assumed Gaussian scatter with a Student's distribution with $10 \mathrm{deg}$ of freedom, which is more robust to the presence of outliers because has more extended tails (Andreon 2012, details how to implement it). We find the same scatter when we restrict our attention to $\log M_{500} / M_{\odot}>14$.

The right-hand panels of Fig. 7 show X-ray luminosity vs. mass, but for the REXCESS (Pratt et al. 2009) cluster sample. This sample is composed of X-ray selected clusters in the range $0.06<z<0.17$. By construction, plotted points tend to be above the fit line because the line represent the scaling relation inclusive of a correction for the X-ray selection function. The XUCS sample, is, as mentioned, X-ray unbiased and largely overlaps REXCESS in redshift $(0.06<z<0.17$ vs.

\footnotetext{
8 The fitting code is distributed in Chapter 8.4 of Andreon \& Waiver (2015), in Andreon \& Hurn (2013) and also available at http://www.brera.mi.astro.it/ andreon/ BayesianMethodsForThePhysicalSciences/model8.4.bug
}

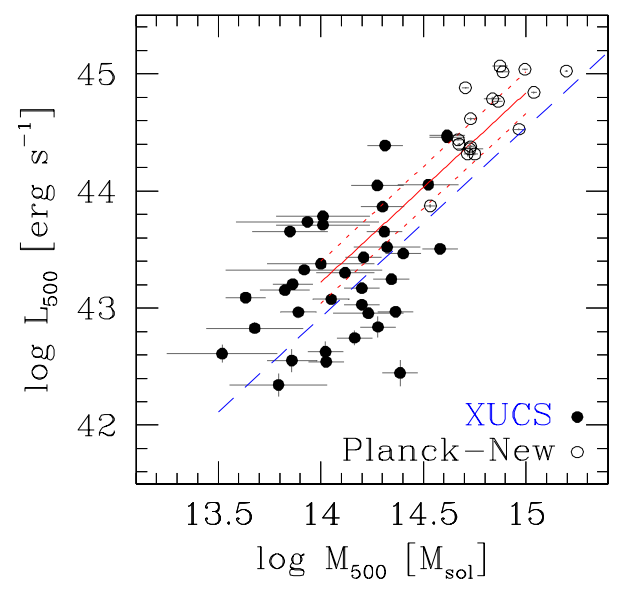

Fig. 8. X-ray luminosity ([0.5-2] keV band) vs. mass for our sample (left-hand panel) and the new Planck clusters (right-hand panel). Masses for Planck clusters are $Y_{\mathrm{X}}$-based, whereas they are caustics based for XUCS. The solid (red) and dotted lines are the REXCESS Malmquist-bias corrected mean and mean \pm the estimated scatter. The long dashed (blue) line is the mean REXCESS scaling offset by 0.3 dex describing the lower envelope of Planck clusters. Several XUCS clusters are below this envelope.

$0.05<z<0.14)$. The mass ranges are also largely overlapping: $70 \%$ of XUCS and $50 \%$ of REXCESS clusters are in the $14<\log M_{500} / M_{\odot}<14.5$ range. While the REXCESS sample tightly obeys the luminosity-mass scaling, the XUCS cluster sample shows a much larger diversity in luminosity at a given mass.

The scatter observed in XUCS is $2.7\left(L_{500}\right)$ and $7\left(L_{500, \text { ce }}\right)$ times the one inferred in REXCESS accounting for the X-ray selection. Therefore, the observed scatter found in our sample cannot be simply explained within the scatter derived accounting for the X-ray selection bias. Rather, with this sample we have unveiled a cluster population exhibiting a larger range of properties for the same mass. This is true also if we consider the $\log M_{500} / M_{\odot}>14$ mass range (see Fig. 7).

The variety seen in XUCS is also larger than that seen by Hicks et al. (2013): the few clusters studied by these authors occupy the low $L_{\mathrm{X}}$ side of the REXCESS distribution, while our clusters go well below. The large variety is indirectly confirmed by Andreon \& Moretti (2011), who observed a 0.5 dex scatter at a given richness, which is known to be a low scatter proxy of mass (e.g., Andreon \& Hurn 2010; Andreon 2015). That sample, however, lacks direct measurements of mass.

\subsection{Is variety correlated to $X$-ray morphology?}

Inspection of the X-ray images of clusters with low X-ray luminosity for their mass do not show any obvious relation between morphology of the X-ray emission and the location of the cluster in the $L_{X}-M_{500}$ plane. For example, CL3000 (see Fig. 3, 0.8 dex below the mean REXCESS relation) is an irregular cluster, and, based on the displacement between X-ray emission and galaxies, an interacting cluster. However, CL1001 shows a similar morphology but it falls close to the REXCESS mean. Furthermore, while CL2007 (see Fig. 2, 1.0 dex below the mean REXCESS relation) is irregular and of low luminosity for its mass, CL1209 (1.0 dex below the mean REXCESS relation) is also of low luminosity but its X-ray appearance is fairly regular (see Fig. 3). Therefore, this suggests no obvious relation between the X-ray morphology and position in the $L_{X}$-mass plane. Paper II will more accurately explore this evidence. 


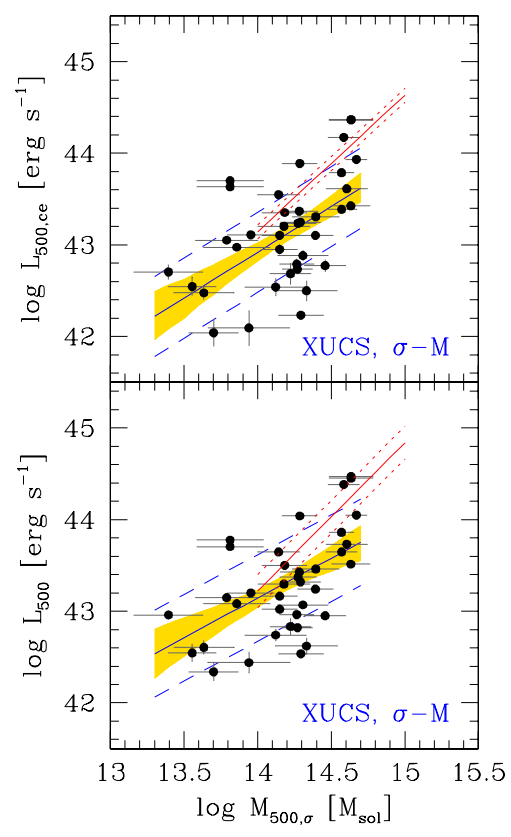

Fig. 9. X-ray luminosity ([0.5-2] keV band) vs. velocity-dispersionbased masses. Top panel: core-excised luminosities. Bottom panel: noncore-excised luminosities. Shading, solid line and dashed corridor are uncertainty on the mean model, mean model, and mean model \pm the intrinsic scatter, respectively.

\subsection{A variety larger than pointed out by Planck?}

Figure 8 shows the $\mathrm{X}$-ray luminosity vs. mass scaling relation for XUCS and for the subsample of new (i.e., not previously known) Planck clusters (Planck Collaboration IX 2011). Masses for Planck cluster are (as REXCESS) based on the pseudopressure parameter $Y_{\mathrm{X}}$ (Kravtsov et al. 2006). The relation defined by the Planck clusters also shows a larger variety than REXCESS (Planck Collaboration IX 2011). While they do not reach the amplitude displayed by this sample, the more extreme point is just 0.3 dex $(<3 \sigma)$ below the best-fit REXCESS relation. In XUCS, CL2081 $\left(\log M_{500} / M_{\odot}=14.4\right)$ is off by $1.4 \mathrm{dex}$, and almost half of the whole sample is more than 0.3 dex fainter than the REXCESS mean (long dashed line). Although the two data sets are sampling complementary parts of the mass function (Planck above, and XUCS below, $\log M_{500} / M_{\odot}=14.5$ ), the variety in the X-ray luminosity at a given mass that we see is larger than observed by Planck, suggesting either that Planck is only unveiling part of the population unveiled by XUCS or that the scatter is mass-dependent. An SZ-selected sample is expected to show a reduced variety of X-ray properties from numerical simulations (Angulo et al. 2012) because the ICM that is emitting in X-ray is also responsible for the inverse Compton effect; this makes the two observables, and selections based on them, correlated.

\subsection{Is variety due to wrong $X$-ray fluxes?}

In this section and in the following sections we investigate possible reasons for the different behaviour of the XUCS and REXCESS samples, starting with the exploration of possible inaccuracies in the $L_{X}$ determination.

In Sect. 3 we show two clusters with similar mass but very different X-ray luminosities. It is very hard to explain a difference, in excess of 900 counts, needed to reconcile the two luminosities, from a faulty data analysis, in particular, when the total number of photons collected in the image of CL2007 is of about 700 , inclusive of background and other sources. In Sect. 4.1 we showed that $L_{X}$ derived by aperture counting is consistent with our more elaborate analysis accounting for excised regions, gaps, variation in exposure time, etc, and that we obtain consistent values using different telescopes. Our $L_{X}$ is also consistent with that derived by REXCESS for the two clusters in common. Finally, a lack of knowledge of the X-ray temperature affects $L_{X}$ to a negligible level $(0.01 \mathrm{dex})$ that is 50 times too small to explain the observed 0.5 dex scatter.

To have overly faint clusters we must have overestimated their backgrounds. A plot of the $L_{X}$ residuals vs. background value shows that this is not the case: overly faint clusters do not have larger values of estimated background, and, furthermore, all XRT background estimates are within $15 \%$, thanks to the stability of the low Swift X-ray background. To further check the background subtraction, we recompute the CL1067 X-ray luminosity using the background from a pointing 31 arcmin away from the cluster. The X-ray luminosity increases by 0.05 dex, 1.5 times the quoted error and ten times smaller than needed to explain the observed $L_{\mathrm{X}}$ scatter at a given $M$. To summarize, we are confident that the cluster X-ray luminosities are precisely measured and cannot be the source of the observed scatter.

\subsection{Is variety due to wrong cluster masses?}

The $L_{X}$-mass scaling explored thus far uses masses derived from caustics, which does not assume the hydrostatic equilibrium nor a relaxed status for the cluster. The noise and bias of caustic masses have been characterized by a few independent teams who use different simulation methods and assumptions. Serra et al. (2011) used hydrodynamic simulations and find a bias of $<5 \%$ at about $r_{200}(\sim 10 \%$ at most radii), uncertainties on individual determinations of about $25 \%$ (at about $r_{200}$ ), and errors in agreement with the uncertainties above. Gifford \& Miller (2013), via $\mathrm{N}$-body simulations, also found a small bias (smaller than $4 \%$ ) and uncertainties on individual determination of about $10-15 \%$. Gifford et al. (2013) confirmed and extended these results with $\mathrm{N}$-body simulations populated by galaxies using different semianalytic prescriptions. Svensmark et al. (2015), using $N$-body simulations, found no bias and a $\sim 15 \%$ scatter. To explain the observed variance in $L_{X}$ at a given mass, caustic masses should have a scatter of $300 \%$ (in addition to the quoted errors).

Observations also indicate that caustic masses cannot be much noisier than their quoted error (i.e., cannot have have understimated errors) because they correlate with almost no scatter with richness (Andreon 2015), which in turns correlates with almost no scatter with weak-lensing mass (Andreon \& Congdon 2014). This latter correlation adopts fixed-aperture masses and, therefore, the source of the tight relation cannot be an effect introduced using mass-related apertures such as $r_{200}$.

In spite of the evidence provided by previous authors that caustic masses and errors are accurate and well understood, and in addition to our accounting of the mass noisiness in our analysis, we want to test our results further by considering masses derived from the galaxy velocity dispersion.

Figure 9 shows the luminosity-mass scaling relation using these masses. By fitting the data we found scaling relation consistent with those measured with caustic masses, namely: for core-excesed luminosities

$\log L_{500, \mathrm{ce}}=(1.00 \pm 0.28)\left(\log M_{500} / M_{\odot}-14.2\right)+43.1 \pm 0.1$, (4) with an intrinsic scatter of $0.44 \pm 0.07$, and for total luminosities $\log L_{500}=(0.85 \pm 0.31)\left(\log M_{500} / M_{\odot}-14.2\right)+43.3 \pm 0.1$, 
with an intrinsic scatter of $0.47 \pm 0.07$. In particular, both scatter estimates are in agreement with those derived with caustic masses, confirming the variety found there.

These X-ray luminosities are still measured within the $r_{500}$ radius derived from caustics. In order to check that the scatter is not due to mismatched apertures, we also adopted radii derived from velocity dispersions (see Sect. 4.2.1). The large variety of $L_{\mathrm{X}}$ values at a given mass is still there. Finally, we checked that using the Munari et al. (2013) relation in place of the Evrard et al. (2008) relation (to convert velocity dispersions in masses) does not affect the scatter at all. Indeed, it can be easily shown mathematically that the scatter is unaffected by whatever velocity dispersions vs. masses relation is used, provided it is a continous function.

To quantify the amplitute of the error on $\sigma_{v}$ needed to explain the scatter of the $L_{X}$-mass relation, let us consider again the clusters CL3013 and CL2007. The slope of the scale relation between core-excised luminosity and mass is 1.49 (Pratt et al. 2009), the slope between mass and velocity dispersion is close to 3 (e.g., Evrard et al. 2008; Munari et al. 2013). Therefore, from scaling relations, the velocity dispersion of CL3013 should be almost twice the velocity dispersion of CL2007 (as shown by the blue solid line in Fig. 5, the velocity distribution that is expected to match the cluster X-ray luminosity). Clearly, this is ruled out by the data.

\subsection{Could be the REXCESS scatter underestimated?}

As already remarked, several works (Planck Collaboration IX 2011; Andreon \& Moretti 2011; Hicks et al. 2013) find a more variegate cluster population than inferred from the REXCESS sample, i.e., that the REXCESS scatter underestimates the total scatter in $L_{X}$ at a given mass. Here we want to focus on a relevant assumption at the base of the mass derivation. Pratt et al. (2009) derive masses from $Y_{\mathrm{X}}=M_{\text {gas }} T$, where the product is largely driven by the change in $M_{\text {gas }}$ because $T$ changes by about $25 \%$ for most of the REXCESS sample, while $M_{\text {gas }}$ variations are of about $100 \%$. The gas mass and $L_{X}$ are derived from the same X-ray photons, in particular, from two closely related radial profiles (of $n_{\mathrm{e}}$ and $n_{\mathrm{e}}^{2}$ ), inducing a covariance between the $Y_{\mathrm{X}}$-derived mass and $L_{\mathrm{X}}$ because of the double use of the same information. In fact, Maughan et al. (2007) find a linear relation between $\log M_{\text {gas }}$ and $\log L_{\mathrm{X}}$ with a negligible (3\%) scatter. Said simply, a cluster brighter/fainter for its mass also has a larger/smaller $Y_{X}$-based mass, moving mostly along the $L_{X}-M_{Y_{X}}$ relation, giving a false impression of a small variance in the REXCESS sample. Since only the orthogonal component of the scatter has been measured and reported by Pratt et al. (2009), the orthogonal scatter underestimated the $L_{X}$ scatter at a given mass.

The above (neglected) covariance is a general feature of the $L_{\mathrm{X}}$ vs. $M_{Y_{\mathrm{X}}}$ analysis, and applies to $Y_{\mathrm{X}}$ based Planck masses in Fig. 6: the variety in the Planck sample may be larger than it appears in Fig. 6. Instead, caustic masses are not derived using $\mathrm{X}$-ray photons. This kind of covariance is absent for our mass$L_{\mathrm{X}}$ determination and our scatter is not underestimated for that reason.

\section{Summary and discussion}

To firmly establish the variety of intracluster medium properties of clusters of a given mass and to break the degeneracies between the parameters of the X-ray cluster scaling relations is important to assemble cluster samples with the following three features: 1) observed in X-ray; 2) with known mass; and; 3 ) whose selection is, at a given cluster mass, independent of the intracluster medium content. Having masses derived independently of the X-ray data is also useful to neglect the covariance induced by the double use of the X-ray photons. The sample, XUCS, presented in this paper offers, for perhaps the first time, an X-ray unbiased view of the cluster population because the cluster sample is assembled independently of the ICM content; at a given mass, clusters are in our sample independent of their X-ray luminosity. It consists of 34 massive clusters $\left(\log M_{500} / M_{\odot}>13.5\right.$ with $70 \%$ of the clusters within $14<$ $\left.\log M_{500} / M_{\odot} \lesssim 14.5\right)$ in the nearby Universe $(0.05<z<0.135)$, observed in X-ray, with abundant spectroscopic data (116 galaxies within the caustics on average), and reliable masses.

XUCS reveals an unexpected variety in X-ray luminosity at a given mass, up to a factor 50. The scatter in X-ray luminosity (total or core-excised) is 0.5 dex. The observed range (and spread) in XUCS is about five times larger than the spread inferred from REXCESS after accounting for the X-ray selection of the latter. We are not just recovering those clusters known to be missed in X-ray selected samples, but a broader population missed altogether by REXCESS. The variety we observe in XUCS also seems larger than in the new Planck cluster sample. This suggests that either Planck is only partially probing the full population seen by XUCS, although the two surveys cover complementary parts of the cluster mass function, or that the scatter in $L_{X}$ at a given mass is mass dependent (larger at lower masses).

Various tests have shown that our X-ray luminosities are reliable and not the cause of the observed variety. Furthermore, by using core-excised X-ray luminosities, we verified that the variety is not due to a central luminosity excess. Masses are derived independently of the X-ray data. In particular, we use caustic masses, which have the advantage of not assuming virial equilibrium nor a relaxed status for the cluster, or velocity dispersionbased masses, which have reliable errors. In both cases, we found the same variety of ICM properties at a given mass.

We known that clusters show a variety in their ICM properties, and in fact there are many individual examples of the effects of AGN feedback (e.g., Voit \& Donanhue 2005), star formation (e.g., Nagai 2006), mergers (Rowley et al. 2004), radiative cooling and other nongravitational effects on the ICM properties. However, the frequency with which these processes occurs in the general cluster population cannot be inferred from an etherogenous collection of clusters and requires an X-ray unbiased sample. In XUCS, we found a scatter of 0.50 dex at a given mass, indicating a much more etherogenous population than previously believed based on X-ray or SZ selected samples. It is left to a future paper to study the implications of the large scatter above on our understanding of the complex ICM physics. Here we just mention that past numerical simulations attempted to infer which physical processes are running in clusters (and the sub-grid physics) by matching slope, intercept, and scatter of observed and simulated scaling relations (e.g., Young et al. 2011). Our larger observed variety prompts some stochasticity (across clusters) of the importance of the different processes, or asks us to enlarge the range of values of parameters addressing the subgrid physics currently kept fixed or bounded in a narrow range (e.g., Pike et al. 2014).

It is believed that X-ray luminosity is a reliable mass proxy with controllable scatter (e.g., Maughan 2007; Pratt et al. 2009), which has important implications for cluster surveys such as Planck and eROSITA, and ultimately for the use of the cluster population for cosmological purposes. At first sight, the variety seen in our sample severely reduces the use of X-ray luminosity, 
including the core-excised one, no longer a mass proxy of moderate scatter. This has an obvious consequence on inferring mass from the observable, and a subdle effect on the knowledge of the selection function, which is central for cosmological tests, of X-ray selected samples. Since the scatter is large, it is hard to characterize the fraction of the population seen by the survey. On the other end, it might be possible to identify subsamples of clusters with a lower variance at a given mass, for example, those with $\log M_{500} / M_{\odot}>14.5$, which may show a smaller spread, as Fig. 8 may suggest, and perform cosmological tests using these. We defer a more detailed discussion to a later paper, when the results of our current X-ray observing campaign on more massive clusters will be completed.

Acknowledgements. A.L.S. acknowledges useful discussions with Antonaldo Diaferio. For the standard SDSS III and GAMA acknowledgements see https://www.sdss3.org/collaboration/boiler-plate.php and http://www.gama-survey.org/pubs/ack.php

\section{References}

Abazajian, K., Adelman-McCarthy, J. K., Agüeros, M. A., et al. 2004, AJ, 128, 502

Abell, G. O., Corwin, H. G., Jr., \& Olowin, R. P. 1989, ApJS, 70, 1

Alam, S., Albareti, F. D., Allende Prieto, C., et al. 2015, ApJS, 219, 12

Andreon, S. 2010, in Bayesian Methods in Cosmology, eds. M. Hobson, A. Jaffe, A. Liddle, P. Mukeherjee, D. Parkinson (New York, Cambridge, UK: Cambridge University Press)

Andreon, S. 2012, A\&A, 546, A6

Andreon, S. 2015, A\&A, 582, A100

Andreon, S., \& Congdon, P. 2014, A\&A, 568, A23

Andreon, S., \& Hurn, M. A. 2010, MNRAS, 404, 1922

Andreon, S., \& Moretti, A. 2011, A\&A, 536, A37

Andreon, S., \& Weaver, B. 2015, Bayesian Methods for the Physical Sciences. Learning from Examples in Astronomy and Physics (Springer)

Andreon, S., de Propris, R., Puddu, E., Giordano, L., \& Quintana, H. 2008, MNRAS, 383, 102

Andreon, S., Trinchieri, G., \& Pizzolato, F. 2011, MNRAS, 412, 2391

Angulo, R. E., Springel, V., White, S. D. M., et al. 2012, MNRAS, 426, 2046

Baldry, I. K., Alpaslan, M., Bauer, A. E., et al. 2014, MNRAS, 441, 2440

Beers, T. C., Flynn, K., \& Gebhardt, K. 1990, AJ, 100, 32

Böhringer, H., Voges, W., Huchra, J. P., et al. 2000, ApJS, 129, 435

Böhringer, H., Schuecker, P., Guzzo, L., et al. 2004, A\&A, 425, 367

Böhringer, H., Schuecker, P., Pratt, G. W., et al. 2007, A\&A, 469, 363
Clowe, D., Gonzalez, A., \& Markevitch, M. 2004, ApJ, 604, 596 Colless, M., Dalton, G., Maddox, S., et al. 2001, MNRAS, 328, 1039 Diaferio, A. 1999, MNRAS, 309, 610

Diaferio, A., \& Geller, M. J. 1997, ApJ, 481, 633

Eckert, D., Molendi, S., \& Paltani, S. 2011, A\&A, 526, A79

Ettori, S. 2015, MNRAS, 446, 2629

Evrard, A. E., Bialek, J., Busha, M., et al. 2008, ApJ, 672, 122

Garmire, G. P., Bautz, M. W., Ford, P. G., Nousek, J. A., \& Ricker, G. R., Jr. 2003, Proc. SPIE, 4851, 28

Gehrels, N., Chincarini, G., Giommi, P., et al., 2004, ApJ, 611, 1005

Gifford, D., \& Miller, C. J. 2013, ApJ, 768, L32

Gifford, D., Miller, C., \& Kern, N. 2013, ApJ, 773, 116

Hicks, A. K., Pratt, G. W., Donahue, M., et al. 2013, MNRAS, 431, 2542

Hill, J. M., \& Oegerle, W. R. 1993, AJ, 106, 831

Jones, D. H., Read, M. A., Saunders, W., et al. 2009, MNRAS, 399, 683

Kravtsov, A. V., Vikhlinin, A., \& Nagai, D. 2006, ApJ, 650, 128

Liske, J., Baldry, I. K., Driver, S. P., et al. 2015, MNRAS, 452, 2087

Maughan, B. J. 2007, ApJ, 668, 772

Maughan, B. J., Giles, P. A., Randall, S. W., Jones, C., \& Forman, W. R. 2012, MNRAS, 421, 1583

Miller, C. J., Krughoff, K. S., Batuski, D. J., \& Hill, J. M. 2002, AJ, 124, 1918

Miller, C. J., Nichol, R. C., Reichart, D., et al. 2005, AJ, 130, 968

Moretti, A., Pagani, C., Cusumano, G., et al. 2009, A\&A, 493, 501

Munari, E., Biviano, A., Borgani, S., Murante, G., \& Fabjan, D. 2013, MNRAS, 430, 2638

Navarro, J. F., Frenk, C. S., \& White, S. D. M. 1997, ApJ, 490, 493

Nagai, D. 2006, ApJ, 650, 538

Pacaud, F., Pierre, M., Adami, C., et al. 2007, MNRAS, 382, 1289

Pike, S. R., Kay, S. T., Newton, R. D. A., Thomas, P. A., \& Jenkins, A. 2014, MNRAS, 445, 1774

Pimbblet, K. A., Smail, I., Edge, A. C., et al. 2006, MNRAS, 366, 645

Planck Collaboration IX. 2011, A\&A, 536, A9

Planck Collaboration XXVII. 2015, A\&A, submitted [arXiv: 1502 .01598]

Planck Collaboration Int. 2012, A\&A, 543, A102

Pratt, G. W., Croston, J. H., Arnaud, M., \& Bohringer, H. 2009, A\&A, 498, 361

Rines, K., Geller, M. J., Diaferio, A., \& Kurtz, M. J. 2013, ApJ, 767, 15

Rosati, P., Borgani, S., \& Norman, C. 2002, ARA\&A, 40, 539

Rowley, D. R., Thomas, P. A., \& Kay, S. T. 2004, MNRAS, 352, 508

Serra, A. L., Diaferio, A., Murante, G., \& Borgani, S. 2011, MNRAS, 412, 800

Stanek, R., Evrard, A. E., Böhringer, H., Schuecker, P., \& Nord, B. 2006, ApJ, 648, 956

Svensmark, J., Wojtak, R., \& Hansen, S. H. 2015, MNRAS, 448, 1644

Turner, M. J. L., Abbey, A., Arnaud, M., et al. 2001, A\&A, 365, L27

Vikhlinin, A., Kravtsov, A., Forman, W., et al. 2006, ApJ, 640, 691

Vikhlinin, A., Burenin, R. A., Ebeling, H., et al. 2009, ApJ, 692, 1033

Voit, G. M. 2005, Rev. Mod. Phys., 77, 207

Voit, G. M., \& Donahue, M. 2005, ApJ, 634, 955

Wojtak, R., Hansen, S. H., \& Hjorth, J. 2011, Nature, 477, 567

Young, O. E., Thomas, P. A., Short, C. J., \& Pearce, F. 2011, MNRAS, 413, 691 